\title{
Apropiación de tecnologías en las redes comunitarias de internet latinoamericanas
}

\section{Technology Appropriation in Latin American Internet Community Networks}

\author{
Mariela Inés Baladron \\ Universidad de Buenos Aires (Argentina)
}

En América Latina se han desarrollado redes comunitarias de internet en diversos territorios como respuesta a la desconexión, pero atendiendo a su vez las problemáticas que atañen a la diferencia y desigualdad de sus comunidades durante la última década. La diversidad de estas experiencias encuentra como punto en común la creación, propiedad y administración colectiva, comunitaria y sin fin de lucro de la infraestructura que despliegan. Este artículo indaga sobre los modos de apropiación que se producen en estas redes, desde la creación tecnológica y los aprendizajes, usos y prácticas adaptadas o creativas que no fueron pensados en el momento de su diseño. Para ello se analiza la creación de tecnología con el LibreRouter, que desarrolló AlterMundi de Argentina; la formación destinada a dar soporte a estas experiencias que brinda el Diplomado Techio Comunitario de Redes AC de México y los usos y prácticas relevados en dos redes comunitarias, QuintanaLibre y Atalaya Sur
In Latin America, internet community networks have been developed in various territories in response to lack of connectivity but also to address those issues that have affected the difference and inequality of their communities over the past decade. The diversity of these experiences shares some common factors: collective, community-serving and non-profit creation, ownership and administration of the infrastructures deployed. This article inquires into how technology appropriation takes place in these networks, from technological creation to adapted or creative ways of learning, using and developing practices that were not initially intended at the time of their design. For this, three cases are analyzed: the creation of technology with LibreRouter developed by AlterMundi in Argentina; the training aimed at supporting these experiences offered by the Diploma course Techio Comunitario by Redes A.C. in Mexico; and the uses and practices surveyed in two community networks, Quintana- 
en Córdoba y Buenos Aires, Argentina, respectivamente. De esta forma, se busca generar conocimiento para tomar en consideración no solo las respuestas que brindan las redes comunitarias de internet en materia de conectividad en América Latina, sino también para la apropiación de tecnologías de sus comunidades.

Palabras clave: redes comunitarias de internet, apropiación de tecnologías, desigualdad, comunicación comunitaria, América Latina.
Libre and Atalaya Sur in Córdoba and Buenos Aires, Argentina, respectively. In this way, this paper seeks to produce knowledge to examine not only the responses provided by Internet community networks in terms of connectivity in Latin America but also to look into the appropriation of technologies in their communities.

Key words: internet community networks, technology appropriation, inequality, community communication, Latin America.

C 1 acceso a internet, a partir de su expansión masiva y comercial desde mediados de la década de 1990, ha sido promovido por su potencial para el desarrollo económico, el ejercicio de la libertad de expresión y como plataforma para el ejercicio de otros derechos humanos por gobiernos y organismos internacionales. Si bien se observa un crecimiento sostenido de la cantidad de usuarios en el mundo, se calcula que casi la mitad de las personas que habitamos el planeta $(46,4 \%)$ aún no utilizaba internet en 2019. En América Latina y el Caribe, los niveles de penetración de internet (37\%) son bajos en relación con Europa $(82,5 \%)$ y más altos que en África $(28,5 \%)$. La llamada "brecha digital" se verifica no solo entre países sino también fronteras adentro, en especial en Latinoamérica. La diferencia entre los principales centros urbanos y las poblaciones rurales ( $\mathrm{y}$ aquellas urbanas o periurbanas en situación de pobreza) presenta índices equivalentes a los que existen entre países denominados por la literatura como "desarrollados" y "en desarrollo": 86\% y 20\%, respectivamente (Gray, Gainous y Wagner, 2016; UIT, 2019).

Las redes comunitarias de internet en América Latina, cuyo antecedente son las redes libres (Sandvig, 2004; Trudel y Tréguer, 2016), crean su propia infraestructura en territorios y poblaciones sin cobertura o donde los servicios del modelo comercial de las telecomunicaciones no son asequibles. Más allá de la diversidad de experiencias, comparten entre sus principios la propiedad y gestión de la infraestructura por parte de la propia comunidad sin fin de lucro, considerando a las personas como la "primera milla" o "primer kilómetro" de las redes. Esta definición apunta a invertir el orden que se plantea en el modelo privado y comercial de prestación de internet, que denomina "última milla" a la red que conecta a los usuarios finales (pueden ser residenciales o corporativos) con la red de las empresas de telecomunicaciones. 
Este artículo propone identificar y caracterizar las prácticas de uso y apropiación de tecnologías surgidas durante el desarrollo de estas experiencias y cómo se vinculan con el modelo de propiedad y gestión comunitaria de infraestructura de estas redes. Los tres casos que se analizan corresponden a avances parciales de la investigación doctoral en curso de la autora y representan tres tipos distintos de apropiación: se trata del desarrollo del LibreRouter como creación tecnológica por parte de AlterMundi de Argentina; el Diplomado de Techio Comunitario impulsado por Redes por la Diversidad, Equidad y Sustentabilidad AC (Redes AC) de México como respuesta a las demandas de formación de las comunidades que acompañan; y, por último, el relevamiento de prácticas creativas de los usuarios de las redes de QuintanaLibre en Córdoba y Atalaya Sur en Ciudad de Buenos Aires. A su vez, el criterio de selección también consideró el reconocimiento interno y externo que suscitan, ${ }^{1}$ la representación rural y urbana y la sostenibilidad en el tiempo de las experiencias.

El abordaje teórico-metodológico propuesto se inscribe en el entrecruzamiento de diferentes perspectivas complementarias entre sí sobre estudios culturales, sociológicos, de comunicación y de género para abordar las diferencias, desigualdades y desconexión contemporáneas y la apropiación de tecnologías. Se privilegia el enfoque cualitativo, que recurre a técnicas de producción primarias con entrevistas en profundidad semiestructuradas a usuarios de las redes comunitarias y observación participante, además de revisión bibliográfica y de documentos producidos por estos actores, junto a fuentes documentales secundarias como notas periodísticas y datos estadísticos para contextualizar estas experiencias.

\section{APROPIACIÓN DE TECNOLOGÍAS EN CONTEXTOS DE DIFERENCIA, DESIGUALDAD Y DESCONEXIÓN}

La idea de "brecha digital" como la separación entre quienes tienen o no acceso a las TIC por razones de disponibilidad geográfica o desigualdades socioeconómicas se ha complejizado en las últimas dos décadas incorporando aspectos como barreras sociales y psicológicas que van más allá de la conectividad física (Deursen y Dijk, 2010). En esta línea surge el concepto de apropiación, que significa más que "adueñarse", es decir, refiere a las prácticas o conjunto de actividades de los sujetos para expresar su vínculo con las tecnologías, "lo que implica la adaptación creativa de las tecnologías a sus propias necesidades, convicciones e intereses, en el marco de la construcción de proyectos de autonomía individual y colectiva" (Morales, 2017: 41), a través de procesos de reflexividad implícitos o explícitos.

1 Cabe mencionar el reconocimiento dentro del colectivo expresado en la I y II Cumbre Latinoamericana de Redes Comunitarias y externo recibido por Internet Society, UIT y el Premio Frida (ediciones 2015 y 2018), entre otros. 
Con el fin de avanzar en el análisis de la apropiación en tanto prácticas, algunos autores han desarrollado una tipología que da cuenta de "un uso distinto $u$ original de la tecnología aprehendida en su dotación de sentido, las que refieren a crear una tecnología distinta en su totalidad o al menos en su justificación, diseño y aplicación a las ya existentes" (Lago-Martínez, Méndez y Gendler, 2017: 78), tanto por parte de individuos, colectivos, corporaciones o gobiernos. Esta tipología reconoce la apropiación reproductiva, la apropiación creativa, la apropiación cooptativa y la creación de tecnologías. En particular, se tomará esta última para analizar el LibreRouter y la apropiación adaptada o creativa de la aplicación de mensajería WhatsApp, cuyo crecimiento ha sido más rápido que el de otras plataformas o redes sociales en los últimos años para el activismo social y político en el mundo (Pang y Woo, 2020), fenómeno que también se observa en las redes comunitarias de internet latinoamericanas.

La problemática de la desconexión en América Latina requiere de un abordaje en conjunto con la diferencia y la desigualdad para incorporar la historia y las temporalidades particulares de la región en los procesos actuales de globalización, interculturalidad y neoliberalismo. Por lo tanto, considerar el mundo desde una mirada conexionista o bajo la metáfora de la red "no elimina las distancias generadas por las diferencias, ni las fracturas y heridas de la desigualdad. El predominio de las redes sobre las estructuras localizadas invisibiliza formas anteriores de mercantilización y explotación —que no desaparecieron- y engendra otras" (GarcíaCanclini, 2004: 79). Las redes comunitarias de internet no solo abordan la falta de conectividad, sino que dicha problemática se imbrica en la complejidad de la diferencia y la desigualdad que atraviesa a los pueblos indígenas y poblaciones rurales o de villas o asentamientos urbanos, por mencionar algunos ejemplos.

El concepto de brecha digital de género también concita especial atención, ya que forma parte de la agenda de organismos internacionales y de las políticas públicas nacionales. Sin embargo, algunos autores critican las mediciones de acceso, frecuencia y tipo de uso de las TIC por parte de hombres y mujeres, ya que lleva implícita una mirada esencialista de género donde las mujeres son consideradas "tecnofóbicas" (Gil-Juárez, et al., 2011; Hilbert, 2011; Gray, Gainous y Wagner, 2016). Por el contrario, sus investigaciones han reconocido que el menor uso y acceso por parte de las mujeres responde de manera directa a condiciones de desigualdad de ingresos, empleo y educación. Por lo tanto, proponen que, en lugar de preguntar por las barreras o problemas a los que se enfrentaron aquellas que están "incluidas", los estudios indaguen sobre los aspectos que las llevaron donde están y a permanecer en dichos entornos, para comprender la configuración de las relaciones entre género, tecnología y sociedad.

En esta dirección, Vergés-Bosch (2012) analiza las motivaciones, los mecanismos posibilitadores y de autoinclusión que despliegan las mujeres en las TIC, desde una perspectiva de los estudios de género. Entre las motivaciones, la autora destaca la autonomía y el placer, que combinan fines utilitaristas y aspectos emocionales. En relación a los posibilitadores, identifica a mentores, entornos amigables o inclusivos y el reconocimiento de sus capacidades, entre otros.

El aprendizaje reviste una particular relevancia en los procesos de apropiación en las TIC. Las categorías propuestas para analizar estos procesos de formación 
son el aprendizaje formal, por medio de la educación reglada; el aprendizaje noformal, "realizado a través de la educación en entidades y/u organizaciones de la sociedad civil en formato de cursos, talleres, conferencias, jornadas y similares"; y el aprendizaje informal, "menos estructurado y estandarizado, más adaptado a cada persona y situación" en contextos cotidianos y vínculos cercanos como familia, amigos, comunidades de práctica o incluso en momentos de ocio (VergésBosch, 2012: 139), que serán retomados para analizar los casos seleccionados.

Finalmente, en la última década las políticas públicas de inclusión digital y universalización de internet han contemplado aspectos como la infraestructura con planes de banda ancha y la entrega de dispositivos a estudiantes y docentes, entre otros (Baladron, 2018). Sin embargo, la articulación con los actores del sector cooperativo, tercer sector o de la economía social y solidaria (entre quienes podría sumarse a las redes comunitarias) todavía es incipiente, aunque también "hacen parte del ecosistema de medios [y] tienen sin embargo mucho para decir y otras formas de hacer" (Monje et al., 2017: 201). Con el fin de analizar los procesos convergentes regulatorios, tecnológicos, de actores o servicios en clave de este tercer sector en América Latina, estos autores proponen el concepto de "convergencia periférica", ya que sin la intervención de una política pública que tome en cuenta las asimetrías es difícil garantizar la supervivencia de actores diversos en un sistema que naturalmente tiende a la concentración y la exclusión.

\title{
LAS REDES COMUNITARIAS DE INTERNET LATINOAMERICANAS
}

\begin{abstract}
Las redes comunitarias son redes de propiedad y gestión colectiva de la comunidad, sin finalidad de lucro y con fines comunitarios.
\end{abstract}

Se constituyen como colectivos, comunidades indígenas u organizaciones de la sociedad civil sin fines de lucro, que ejercen su derecho a la comunicación, bajo principios de participación democrática de sus miembros, equidad, igualdad de género, diversidad y pluralidad.

La información sobre el diseño y funcionamiento es abierta y accesible, permitiendo y favoreciendo la extensión de la red por parte de los usuarios. Las redes comunitarias fomentan los servicios y contenidos locales, promueven la neutralidad de la red y la celebración de acuerdos de interconexión y tránsito libres y gratuitos con las redes que ofrecen reciprocidad.

Documento final de la I Cumbre Latinoamericana de Redes Comunitarias, $2018^{2}$

Esta definición de red comunitaria de internet menciona numerosos aspectos que refieren tanto a factores identitarios como a características de estas experiencias en América Latina que interpelan sobre las formas de pertenencia, posesión y

2 Recuperado el 1 de agosto de 2019 de: <http://cnsig.info/assets/documento-final CLRC-2018.pdf>. 
64 participación de los recursos de conectividad que desarrollan. Por lo tanto, se evidencian aspectos poco frecuentes en el momento de definir una infraestructura de red: menciones a diferencias étnicas y de género de las personas que las crean y utilizan; la transparencia y apertura sobre sus características técnicas; el ejercicio del derecho a la comunicación sin fin de lucro como principal objetivo; y una forma de propiedad y gestión democrática y colectiva. Si bien muchos de estos aspectos son principios que se persiguen, otros tantos son características que describen a estas experiencias tal como funcionan en el día a día en sus territorios.

La diversidad latinoamericana se expresa en las organizaciones y redes que formaron parte de la I y II Cumbre Latinoamericana de Redes Comunitarias, celebradas en Argentina en 2018 y en Colombia en 2019, donde participaron representantes de esos países, así como también de Brasil, Cuba, Ecuador, México y Nicaragua. Al pensar la desconexión junto a la diferencia y la desigualdad y las distintas temporalidades latinoamericanas (García-Canclini, 2004), observamos que las redes comunitarias y las organizaciones que las promueven trabajan con comunidades indígenas, campesinas, afrodescendientes, migrantes, por mencionar algunas, que según los territorios donde se emplazan a veces se enfrentan a conflictos políticos y/o de violencia, además de a situaciones de precarización y vulnerabilidad socioeconómica.

En esta diversidad de realidades se ha alcanzado una agenda con objetivos y demandas comunes, además de una definición consensuada de red comunitaria de internet, también expresados en la declaración de la I Cumbre Latinoamericana de Redes Comunitarias. Como actores de la convergencia periférica (Monje et al., 2017), demandan reconocimiento legal como prestadores sin fin de lucro y sin discriminación; acceder a Fondos del Servicio Universal ${ }^{3}$ y el diseño e implementación de regulaciones y políticas públicas que den respuesta a las necesidades específicas que plantean.

\section{LIBREROUTER: UN 'HARDWARE’ LIBRE Y ABIERTO}

Una de las principales dificultades a las que se enfrenta una comunidad para desarrollar su propia infraestructura consiste en tener entre sus miembros a personas con el conocimiento necesario para instalar y mantener la red, además del capital inicial para adquirir el equipamiento. AlterMundi es una asociación civil basada en la Argentina que trabaja para facilitar el nacimiento de redes libres y comunitarias desde una lógica colaborativa y mediante el uso y desarrollo de hardware y software libre para "un modelo de red de autoprovisión de servicios de

3 Los Fondos del Servicio Universal fueron creados en muchos países durante los procesos de privatización de los monopolios estatales de las empresas de telefonía a principios de la década de 1990 y apuntan a reducir la brecha digital generada por el modelo privado comercial de las telecomunicaciones y las TIC. 
comunicaciones que pueda ser replicable en regiones digitalmente excluidas". ${ }^{4}$ Este modelo promueve las redes Mesh, que se caracterizan por una arquitectura que en lugar de conectar cada punto Wi-Fi con una red cableada comunica el tráfico entre dispositivos de los cuales solo uno está conectado a internet; esta decisión tecnológico-política propone que la red sea descentralizada.

El despliegue de redes comunitarias se realiza con equipos que no han sido diseñados específicamente para redes Mesh, sino para el mercado de los pequeños ISP (Internet Service Provider). A partir de 2015 se sumó una nueva limitación: los fabricantes de hardware modificaron sus routers de manera que no puedan utilizar software libre en cumplimiento de nuevas regulaciones de Estados Unidos. ${ }^{5}$ Con el objetivo de encontrar una solución a esta problemática, AlterMundi se propuso desarrollar un hardware abierto, el LibreRouter. Esta tecnología buscaba responder a la arquitectura de las redes Mesh, simplificar los procesos de instalación y mantenimiento de las redes, mejorar la calidad de las conexiones y bajar el costo del equipamiento.

Este proyecto logró financiamiento a través del Premio Fondo Regional para la Innovación Digital en América Latina y el Caribe (FRIDA) 2015 de LACNIC 6 como reconocimiento al trabajo de AlterMundi con el impulso de la red comunitaria QuintanaLibre en el Valle de Paravachasca, en la provincia de Córdoba, Argentina. Esta red fue creada en 2012 en San José de La Quintana, un pueblo de alrededor de 1.000 habitantes, que luego se extendió a otros seis lugares de la zona (San Isidro, Anisacate, Valle de Anisacate, La Bolsa, La Serranita y Nono). Se trata de una red Mesh con 70 nodos, que brindan acceso a alrededor de 280 equipos, que se mantiene con trabajo voluntario y un aporte mensual solidario de sus usuarios. También recibieron subvenciones para desarrollar el modelo y experimentar cambios en la red, que a su vez provee de acceso a internet a tres escuelas, espacios públicos y centros culturales locales (Belli, 2017).

A partir de la tipología propuesta por Lago-Martínez, Méndez y Gendler (2017), el LibreRouter consiste en la creación de tecnología propia y distinta, basada en saberes y conocimiento de carácter técnico, científico y práctico, surgido en un contexto específico para dar respuesta a restricciones regulatorias y económicas, como una opción que se adapta mejor en rendimiento para los usos

4 Este texto fue recuperado del cuadernillo Redes libres, comunitarias y descentralizadas producido por AlterMundi y disponible en su sitio (última consulta: 3 de enero de 2020): $<$ http://docs.altermundi.net/>.

5 El organismo regulador de ese país, la FCC- Federal Communications Commission, modificó las reglas para el uso de equipamiento sobre espectro no licenciado que opera en la banda de $5 \mathrm{GHz}$, con el fin de evitar que se utilicen otras frecuencias. En respuesta a estos cambios, los fabricantes decidieron directamente cerrar sus equipos, lo que generó un nuevo obstáculo para las redes comunitarias que utilizan software libre.

6 El Registro de Direcciones de Internet para América Latina y Caribe (LACNIC) es la organización responsable de la asignación y administración de los recursos críticos de internet para la región. 
de las redes comunitarias. A su vez, el LibreRouter coincide con la categoría de tecnología "social" porque fue elaborada de forma colaborativa y grupal con la motivación de que estos equipos "puedan usarse para mejorar la calidad de vida o desenvolvimiento social, económico y cultural de sus potenciales usuarios. La meta es lograr un financiamiento autogestivo del grupo creador y generar una mejora o ayuda para sus usuarios" (Gendler et al., 2018: 54).

A comienzos de 2018 AlterMundi realizó las primeras pruebas del prototipo y en junio de ese año empezó su fabricación a través de una empresa de pequeña escala que produce hardware abierto en China. El LibreRouter aporta ventajas de índole técnico y económico: incluye todos los elementos necesarios para instalar un nodo completo (caja preparada para estar a la intemperie, cables y antenas) y su costo es aproximadamente tres veces menor a un router comercial, además de ofrecer estabilidad, según explica Nicolás Echániz, referente de AlterMundi, ya que "a veces se discontinuaba un equipo y las comunidades dependían de un modelo específico y complicaba mucho encontrar un hardware que sirva, es un problema histórico" (comunicación personal). ${ }^{7}$

En paralelo al proceso de fabricación del LibreRouter, AlterMundi continuó el trabajo correspondiente al software y la documentación técnica para liberar el dispositivo y que su fabricación pueda replicarse. En 2019 el principal avance en cuanto a software fue la autoconfiguración de los equipos al momento de su instalación, para facilitar el proceso. El siguiente paso fue el desarrollo de un sistema de soporte remoto para reforzar la autonomía en el mantenimiento de las redes:

La idea es que las comunidades puedan organizarse mejor internamente para ir llevando registro de los problemas y acciones para solucionarlos, el estado de sus redes y dar acceso externo al equipo de soporte, con un permiso que dan los administradores de cada red, con la posibilidad de elevar un problema, incidencia o reporte que no pueden solucionar internamente (Nicolás Echániz, comunicación personal).

Estas prácticas de creación de tecnología se complementan con talleres y semilleros para la formación y capacitación en la instalación de nodos y mantenimiento técnico de las redes comunitarias. A partir de la sistematización de esas experiencias, AlterMundi ha elaborado cuadernillos con ilustraciones y videos para guiar la instalación técnica, mapear y planificar una red y aportar conceptos sobre redes libres, comunitarias y centralizadas como material de aprendizaje para futuros despliegues. Los cuadernillos están disponibles en su sitio web y atravesaron un largo proceso de revisión con otras organizaciones de la región para adaptar los diferentes léxicos del español entre países latinoamericanos, además de traducirlos al portugués e inglés y ponerlos a prueba en "terreno". De esta forma, se producen instancias no-formales de aprendizaje que articulan recursos variados: talleres, cuadernillos, material gráfico, audiovisual, medios de consulta virtuales, etc.

7 Entrevista personal realizada el 30 de noviembre de 2019 en la I Cumbre Argentina de Redes Comunitarias realizada en La Serranita, provincia de Córdoba. 


\section{Imagen 1. LibreRouter}

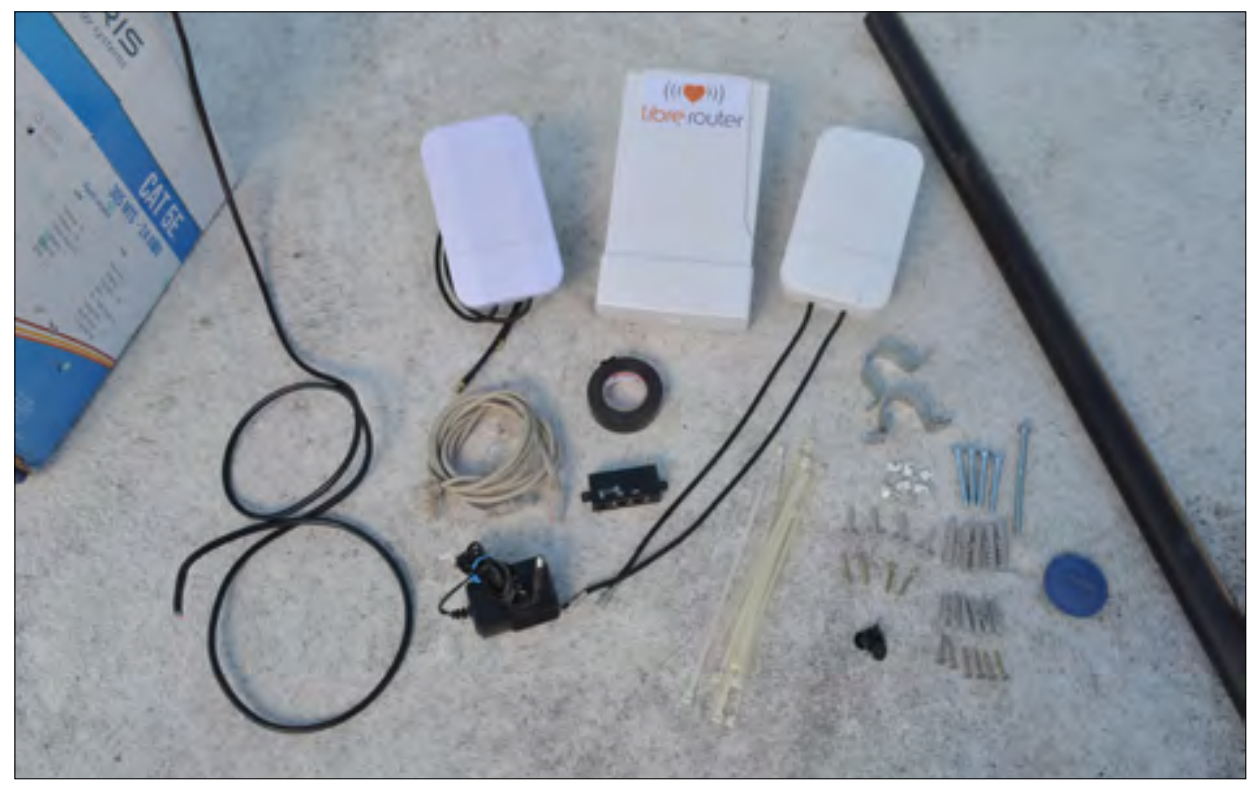

Crédito: AlterMundi.

\section{TECHIO COMUNITARIO, UN DIPLOMADO PARA FORTALECER LA APROPIACIÓN DE TECNOLOGÍAS}

El Diplomado Comunitario de Promotores en Telecomunicaciones y Radiodifusión "Techio Comunitario" es el resultado de un proceso que comenzó en 2012, a partir de identificar las necesidades de formación técnica de comunidades indígenas para desarrollar sus proyectos de radiodifusión, telefonía y redes comunitarias. Entre octubre de 2016 y mayo de 2017 se realizó la primera edición, en la que participaron 36 personas de 6 estados de México, coordinada por Redes AC y Palabra Radio, de donde participaron otras 17 organizaciones. Una segunda generación tuvo lugar entre 2018 y 2019 y la tercera estaba en desarrollo al cierre de este artículo.

Redes AC trabaja desde 2004 para la promoción de la comunicación indígena comunitaria, a través de programas dirigidos a la incidencia política y regulatoria para un marco legal sin discriminación, espacios de formación de comunicadores indígenas, redes comunitarias para el desarrollo de telecomunicaciones indígenas e investigación aplicada. Junto a Tecnologías Indígenas Comunitarias (TIC AC) y Rhizomática han desarrollado redes de telefonía celular comunitaria. En julio de 2016 se logró la primera concesión social indígena para administrar y operar redes de telecomunicaciones y radiodifusión autónomas (también de telefonía celular) en México; en 2019 conectaban aproximadamente a 4.000 usuarios en más de 70 localidades indígenas y rurales, con un modelo de sostenibilidad que in- 
cluye la organización cooperativa y la toma de decisiones asamblearia, un aporte económico mensual de los usuarios y la formación y capacitación técnica. ${ }^{8}$

Los contenidos del Diplomado responden a la necesidad de formación técnica planteada por parte las comunidades y organizaciones con las que trabajan; Daniela Parra Hinojosa, integrante de Redes AC desde sus inicios, explica que el objetivo fue "romper este ciclo de dependencia que existía para la reparación, mantenimiento y sostenibilidad de equipos, e instalaciones de infraestructura" (comunicación personal). ${ }^{9}$ Para ello se diseñó un Tronco Común para el aprendizaje básico de comunicación comunitaria y tecnologías, electricidad, electrónica y software libre, como conocimiento previo para avanzar luego en las espacialidades: radiodifusión, telefonía celular comunitaria y redes inalámbricas de internet. Por último, un módulo de integración relaciona el conocimiento técnico con los procesos de comunicación comunitaria, tomando en cuenta aspectos jurídicos y económicos (Huerta y Baca, 2018). Este programa integra la visión indígena, elementos de la educación popular y planteamientos de las sociedades de conocimiento libre.

El Diplomado podría identificarse como una instancia de aprendizaje no-formal (Vergés-Bosch, 2012), ya que la coordinación está a cargo de organizaciones de la sociedad civil y el formato educativo que propone incluye talleres y laboratorios, un esquema más flexible que la educación formal o reglada (también por la duración en el tiempo), y su diseño y desarrollo contemplan las necesidades específicas planteadas por las comunidades y su cosmovisión, adaptados a sus principios y formas de aprendizaje.

Un aspecto fundamental para lograr que esta formación sea un aporte en la apropiación de tecnología es la metodología, que consiste en la investigación acción participativa, cruzados por la educación popular y la pedagogía popular. Al respecto, Huerta y Baca (2018) explican que esta metodología retoma la idea de praxis integrada a la reflexión como forma de producción de conocimiento de la visión indígena, ya que dichas instancias no están "separadas". A su vez, "la compartencia" o socialización de conocimiento ha sido incluida en el Diplomado a través de laboratorios técnicos y una plataforma online, para que el proceso de formación tenga continuidad y refuerce esos espacios del compartir en el desarrollo de los proyectos en las comunidades.

Por último, las guías de los talleres son un cierre temporal o parcial de estos procesos y, al igual que AlterMundi, Redes AC publica esos documentos en su sitio web para que sean de consulta abierta:

Abrimos estas guías para que más personas conozcan la metodología pero también puedan nutrirla, cuestionarla y adaptarla a sus necesidades. Una de las bondades de la

8 Estos datos surgen “Telecomunicaciones Indígenas Comunitarias 'conecta' a 4.000 usuarios en más de 70 localidades", DPL News, <https://digitalpolicylaw.com/telecomunicacionesindigenas-comunitarias-conecta-a-4-mil-usuarios-en-mas-de-70-localidades/> (última consulta: 10 de diciembre de 2019).

9 Entrevista realizada en Buenos Aires el 28 de noviembre de 2019. 
mirada que hemos elegido tiene que ver con hacer preguntas clave que no indican caminos en específico, sino que la gente misma es la que va decidiendo por dónde quiere caminar, pero que esas preguntas sí detonen un montón de reflexiones interesantes. Nosotros, pues, no vamos con ninguna respuesta predeterminada a las cosas que vamos viendo en los talleres, sino que vamos construyendo con la misma gente (Parra Hinojosa, comunicación personal).

Esta formación ha sido desarrollada no solo para sortear la desconexión, sino desde el reconocimiento de la diferencia tal como plantea García-Canclini (2004), donde la visión indígena de producción de conocimiento y sus temporalidades son incorporadas. En lugar de identificar "barreras" para la apropiación, el Diplomado construye un aprendizaje más horizontal y reflexivo de la configuración de los sujetos con la tecnología, que comienza por identificar sus motivaciones y demandas para acompañarlas con mecanismos posibilitadores y de autoinclusión que se construyen en conjunto (Gil-Juárez et al., 2011; Vergés-Bosch, 2012).

\section{Imagen 2. Taller en el marco del Diplomado Techio Comunitario}

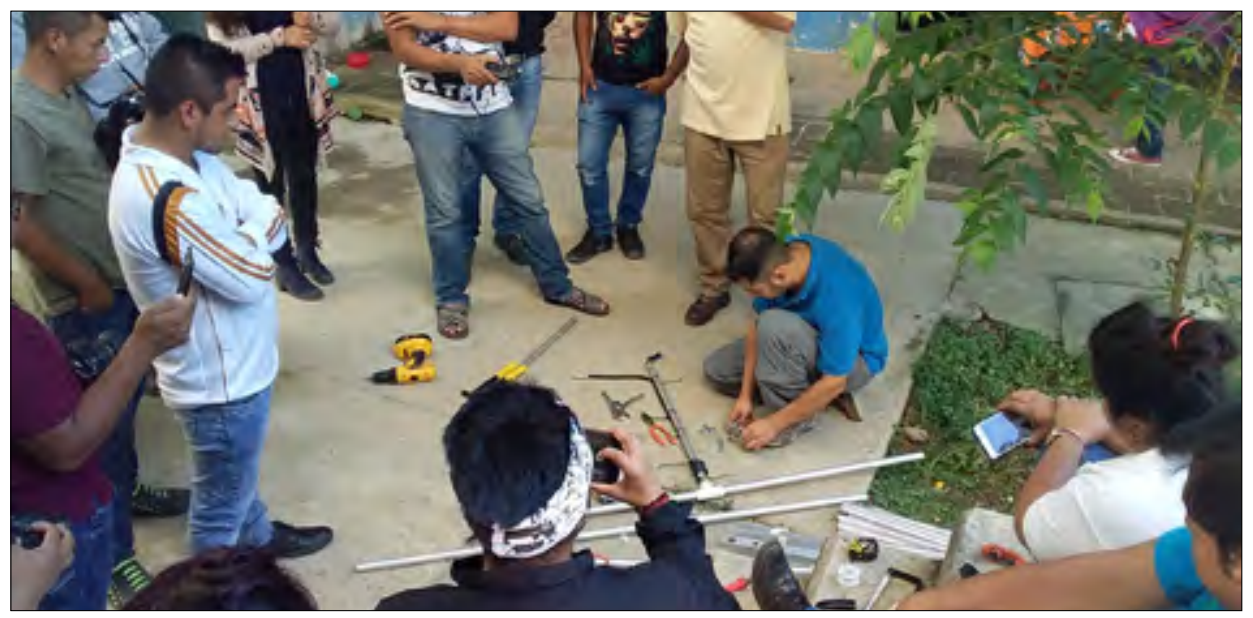

Crédito: Redes AC.

\section{USOS Y PRÁCTICAS EN LAS REDES COMUNITARIAS DE ATALAYA SUR Y QUINTANA LIBRE}

Las redes comunitarias de internet también habilitan aprendizajes, usos y prácticas inesperadas de la tecnología, más allá de los propósitos imaginados en el momento de su desarrollo. Siguiendo a Lago-Martínez, Méndez y Gendler (2017: 79), se trata de apropiación adaptada o creativa, ya que remiten a una tecnología existente pero se generan "nuevas y originales formas de uso y aplicación de las mismas" en lugar de aquellos planificados en el momento de su diseño. 
A partir de la observación y entrevistas semiestructuradas realizadas a integrantes de las redes de Atalaya Sur en Villa 20, un barrio popular de la Ciudad de Buenos Aires, y QuintanaLibre, en José de la Quintana, Córdoba, se sistematizan dos ejemplos: el uso de WhatsApp y los talleres de capacitación, respectivamente.

\section{Atalaya Sur y LA INSERción De LA RED EN UN BARRIO URBANO}

Proyecto Comunidad desarrolló a partir de 2014 la primera experiencia de distribución no comercial de internet en Villa 20 a través de la red de Atalaya Sur con puntos Wi-Fi de acceso abierto y gratuito. A partir de 2018 comenzó en paralelo el despliegue de una red cableada que brinda servicio domiciliario a alrededor de 30 familias, con el pago de una cuota mensual solidaria. El trabajo territorial en Villa 20 incluye un merendero, talleres y capacitaciones, microcréditos para emprendedores, entre otros. El acercamiento a la tecnología comenzó con el dictado de talleres de robótica para niños y los vecinos plantearon la necesidad de tener internet.

Un aspecto fundamental para analizar las redes comunitarias corresponde al lugar de su emplazamiento, ya que en muchos casos influye en las posibilidades de acceso a servicios, lo que profundiza aún más las desigualdades socioeconómicas. Los datos relevados por el gobierno local en el censo realizado en mayo de 2016 por el Instituto de Vivienda ${ }^{10}$ indican que en Villa 20 viven 27.990 personas en 4.559 viviendas, de las cuales el $45 \%$ tiene un estado de construcción regular o malo y la tasa de hacinamiento crítico es del $14,3 \%$. Con respecto a los servicios, el $4 \%$ accede a electricidad, el $25 \%$ a cloacas y el $35 \%$ a agua potable. Los indicadores de precarización laboral, salud y educación demuestran una marcada desigualdad respecto de otros barrios de la Ciudad de Buenos Aires: en los asentamientos el $76 \%$ de la fuerza laboral se encuentra precarizada, mientras que en el resto de la ciudad ese indicador corresponde al 40\%, a lo que se suman la estigmatización y discriminación que sufren (Motta y Almansi, 2017 y Díaz, 2019).

Esta desigualdad también se observa en relación con los servicios TIC; Villa 20 se ubica en la jurisdicción con los indicadores más altos de conectividad del país. Sin embargo, la oferta de servicios comerciales corresponde a tres empresas tercerizadas o franquicias, con menor calidad y mayor costo que en otros barrios. A partir de un relevamiento propio a partir de entrevistas, información web de las empresas y visitas al barrio, se pudo obtener la siguiente información: en junio de 2019 el costo mensual de $1 \mathrm{Mb}$ de internet domiciliaria comercial era equivalente a 11 dólares o 9,6 euros en Villa 20 (\$480 pesos argentinos), mientras que en el resto de la Ciudad podían contratarse $50 \mathrm{Mb}$, televisión de pago y telefonía fija (triple play) por 38 dólares o 33 euros ( $\$ 1654$ pesos argentinos), lo que indicaría que $1 \mathrm{Mb}$ era equivalente a 0,76 dólares o 0,66 euros, además de incluir otros servicios. ${ }^{11}$

10 Disponible en: <https://www.estadisticaciudad.gob.ar/eyc/>.

11 Para estos cálculos se tomó en consideración la cotización del peso argentino con el dólar estadounidense y el euro correspondientes a la fecha en que se realizó el relevamiento, 30 de junio de 2019. 
Graciela González Jara es referente de Proyecto Comunidad en Villa 20, donde vive hace más de treinta años. Cuando compara la red de Atalaya Sur con los servicios comerciales de internet en el barrio reconoce dos diferencias clave: la cuota solidaria es más económica y el servicio técnico y las reparaciones son resueltas por los propios vecinos, mientras que los otros proveedores pueden demorar semanas. "Cuando viene una empresa y provee un servicio vos te desentendés: 'listo, tengo internet, ya está'. No sé cómo funciona ni de dónde viene. Esta experiencia es totalmente distinta: sabés de dónde viene, cómo funciona y aprendés del mantenimiento" (comunicación personal). ${ }^{12}$

La propiedad y gestión comunitaria de la infraestructura de la red promueven el aprendizaje sobre cómo funciona internet y algunas habilidades básicas para identificar el foco del problema cuando el servicio no funciona. Una herramienta que surge de las entrevistas realizadas es WhatsApp: los integrantes de la red tienen un grupo que funciona como espacio de consulta sobre temas técnicos, para coordinar las reuniones mensuales presenciales de gestión de la red y de toma de decisiones, principalmente. Tal como plantean Pang y Woo (2020), la aplicación de mensajería cumple el rol de facilitar las relaciones e interacciones de comunicación personalizada dado que ofrece un espacio cerrado de intercambio entre personas identificadas.

\section{Imagen 3. Enlace inalámbrico en Villa 20, CABA, de la red Atalaya Sur}

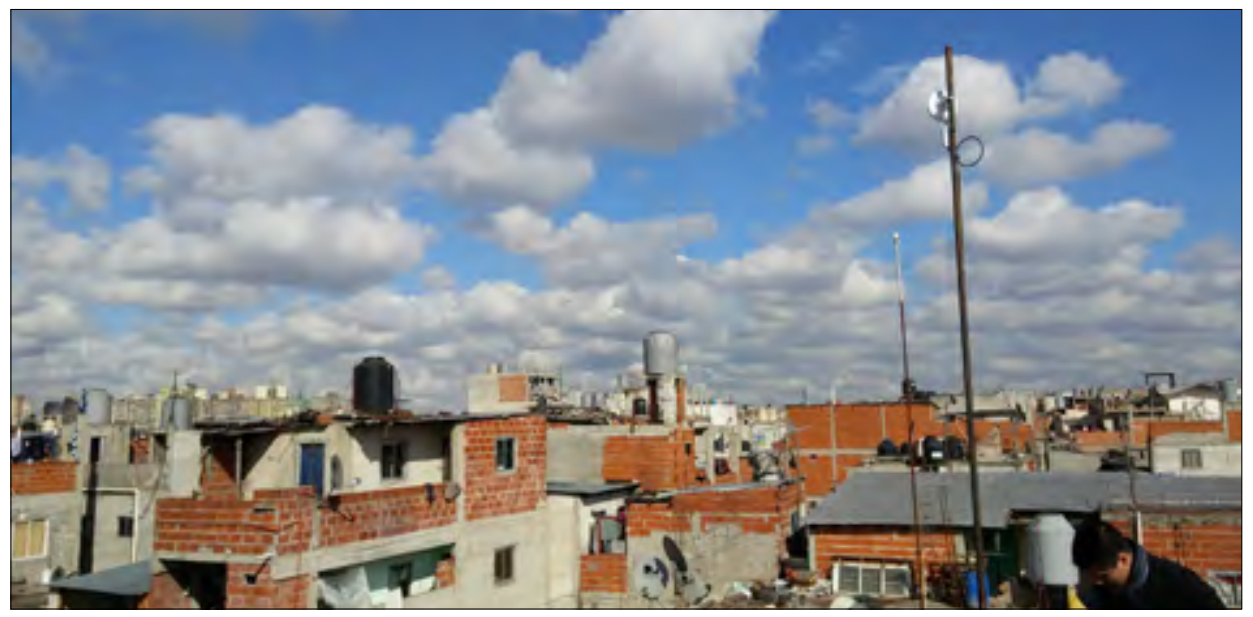

Crédito: Atalaya Sur.

Por último, un aspecto que los autores también advierten sobre WhatsApp es su potencial uso para diseminar noticias falsas con mayor eficacia, ya que las personas las reciben a través de contactos o grupos en los que deposita altos niveles de

12 Entrevista personal realizada en el local de Proyecto Comunidad en Villa 20 el 28 de junio de 2019. 
credibilidad en comparación con otras redes sociales. Una anécdota presenta otra apropiación adaptada o creativa de los grupos de WhatsApp en el barrio en relación con este fenómeno. El domingo 16 de junio de 2019 tuvo lugar un apagón masivo que afectó a casi la totalidad del territorio argentino y algunos países limítrofes por alrededor de 6 horas. Durante ese tiempo de incertidumbre empezaron a circular audios y videos falsos que indicaban que había colapsado la represa de Yacyretá (ubicada en el límite entre Argentina, Brasil y Paraguay) y que en unas horas todo el país estaría bajo el agua. Estos mensajes llegaron a los grupos de los vecinos y Graciela González Jara relata que en esa ocasión, así como en otras, se pide que la información sea chequeada antes de compartirla y evitar el pánico:

El teléfono, internet y la red te dan la posibilidad de calmar a esta gente; entré a los principales portales de noticias y les mandaba los links para que vieran que la noticia era falsa. Si no contestaba, todas las personas se quedaban con esa versión de los hechos, con miedo, con pánico de ver esas imágenes de una represa (...) Tener otro canal de información sirve muchísimo y también enseñarle al vecino que busque otra versión, que chequee, y si lo quiere compartir, aún más (comunicación personal).

De esta forma, el grupo de WhatsApp no solo habilita un canal de gestión técnico y administrativo de la red, sino que permite la articulación de acciones e información para atender otras problemáticas de los vecinos en un entorno de fuertes desigualdades sociales en el que el aprendizaje informal es potenciado en estos intercambios diarios.

\section{LAS MUJERES DE LA RED QUINTANALIBRE}

Las personas más activas en las tareas del mantenimiento de la infraestructura de la red de QuintanaLibre ${ }^{13}$ son mujeres que no tenían formación técnica previa. Sonia Martínez vive en José de la Quintana hace más de veinte años y se sumó a la red hace seis; en la actualidad dicta talleres para el armado de nodos. Por las características de las ocupaciones y conformación de las familias del pueblo, el rol de las mujeres se fue consolidando:

De los 68 nodos de QuintanaLibre, 15 son de mujeres solas que somos jefa de hogar, no tenemos un hombre al lado, marido o hijo que nos ayude. Desde que empecé con QuintanaLibre o pagaba o aprendía. Y hemos hecho que las demás mujeres se involucren sin esperar que vuelva el hombre a la casa. En esta zona muchos hombres se van a trabajar y se van mucho tiempo, 15 días o más, porque son mineros o trabajan en líneas de alta tensión eléctrica. A mí me salió naturalmente, recién ahora estoy reflexionando que lo que hago es importante y cuál es la importancia de todo esto (comunicación personal). ${ }^{14}$

13 Las características de esta red fueron presentadas en el apartado sobre LibreRouter.

14 Entrevista realizada el 26 de septiembre de 2019 en Fusagasugá, Colombia, durante la $2^{\underline{a}}$ Cumbre Latinoamericana de Redes Comunitarias. 
En el relato de Sonia observamos varias de las motivaciones, mecanismos posibilitadores y de autoinclusión de las mujeres en las TIC que identifica Vergés-Bosch (2012). En primer lugar la autonomía, que habitualmente combina el placer del aprendizaje de tecnologías y un fin utilitarista, como evitar pagar por un servicio. En ese proceso, las mujeres reconocen sus propias capacidades, habitualmente reconocidas solo en otros (marido, hijo, etc.), además de desarrollar un espacio horizontal y colectivo con sus pares y resolver los requerimientos de su entorno. También la idea de reflexividad sobre las prácticas del sujeto y la tecnología con un fin social o político se hace presente, con una valoración de ese proceso.

El reconocimiento de los mentores es otro punto que aparece en las entrevistas; allí se identifica a Nicolás Echániz y Jesica Giudice de AlterMundi (quienes también viven en José de la Quintana e impulsaron la creación de la red) como las personas que apoyaron el aprendizaje técnico y son identificados como fuentes de consulta ante problemas técnicos más complejos.

Otros mecanismos que describe Vergés-Bosch (2012) en la autoinclusión de las mujeres en las TIC son el emprendedurismo y la transversalidad de las TIC en sus tareas. En esa línea, Sonia Martínez describe un proyecto que pretende desarrollar con un grupo de mujeres de la red, La Fabrikita, en articulación con el desarrollo del LibreRouter de AlterMundi. La propuesta es fabricar parte del equipamiento que actualmente se realiza en China (caja, antenas, etc.) en José de La Quintana. "Es un proyecto femenino para el empoderamiento femenino ya que en esta zona es bastante escaso el trabajo para las mujeres y es una forma de crear trabajo" (comunicación personal).

En este caso, el soporte técnico de los nodos de una red comunitaria y los talleres de capacitación no fueron concebidos particularmente con una mirada de género o para las mujeres que no tenían formación previa en tecnologías de una forma explícita; sin embargo, ellas descubrieron sus motivaciones, construyeron confianza en sus capacidades y realizaron aprendizajes que convierten a estas prácticas en apropiación de tecnología, además de promover nuevos proyectos vinculados al LibreRouter.

\section{REFLEXIONES FINALES}

La propiedad y gestión colectiva de los recursos de conectividad con una lógica sin fin de lucro por parte de las redes comunitarias latinoamericanas implican la participación de los usuarios en el diseño, tendido y mantenimiento de la red. En la mayoría de los casos, las comunidades donde se desarrollan estas redes no cuentan con personas con conocimientos técnicos específicos para el montaje y mantenimiento de redes; por ello, el aprendizaje y las prácticas de apropiación de tecnología son un factor fundamental en estos procesos.

A partir de los casos analizados, observamos que el LibreRouter como ejemplo de creación de tecnología está imbricado con el modelo de propiedad y gestión comunitaria de la infraestructura. Esto se expresa tanto en las motivaciones para su desarrollo — solucionar la falta de disponibilidad de equipamiento- como en los objetivos que persigue: reforzar el modelo horizontal y descentralizado que 
promueven las redes Mesh; bajar el costo en equipos para el despliegue de redes sin fines de lucro; y generar talleres y materiales de apoyo que promueven el aprendizaje y apropiación de la tecnología por las comunidades que no cuentan con conocimientos técnicos especializados, para lograr autonomía en la creación y mantenimiento de sus redes.

Por su parte, el Diplomado Techio Comunitario como instancia de aprendizaje no-formal no solo surgió a partir de las demandas de las propias comunidades sobre sus necesidades de formación técnica, sino que sus contenidos y metodología incorporan la visión indígena, sus formas de aprendizaje y socialización del conocimiento. De esta forma, la desconexión y la diferencia son trabajadas de forma conjunta, respetando también la temporalidad y los procesos de las comunidades indígenas, ya que el objetivo es la promoción de la comunicación indígena a través de la radiodifusión, la telefonía y redes comunitarias.

Por último, observamos el surgimiento de usos y apropiaciones creativas durante el desarrollo de estas experiencias. Los integrantes de la red de Atalaya Sur comparten información relevante sobre las desigualdades que viven en su barrio y generan aprendizajes informales sobre la circulación de noticias falsas a través de WhatsApp, mientras que la red de QuintanaLibre generó un entorno de autoinclusión en las TIC para las mujeres del pueblo.

Las redes comunitarias de internet de América Latina, además de constituirse como una opción para llevar conectividad a poblaciones en zonas rurales o en situación de pobreza, pueden generar condiciones para la apropiación de tecnología en sus comunidades con un abordaje que atiende las situaciones de desconexión, desigualdad y diferencia de forma conjunta. Continuar la producción de conocimiento en esta línea pretende generar aportes para poner en consideración la articulación de estas experiencias con el conjunto de actores del sector y su promoción a través de políticas públicas.

Mariela Inés Baladron (mariela.baladron@ gmail.com). Doctoranda en Ciencias Sociales de la Universidad de Buenos Aires (UBA), magíster en Industrias Culturales: Políticas y Gestión por la Universidad Nacional de Quilmes (UNQ) y licenciada en Ciencias de la Comunicación Social (UBA). Ha obtenido la beca de investigación y formación UBACyT y el Fondo Semilla para el Fomento de la Investigación de Posgrado por su proyecto de doctorado. Es investigadora y miembro del Comité Académico del Instituto de Estudios de América Latina y el Caribe (IEALC) e integra el Observatorio Comunicación y Derechos (DERCOM), de la Facultad de Ciencias Sociales de la UBA. Es docente de las asignaturas Políticas y Planificación de la Comunicación y Derecho a la Información de la carrera de Ciencias de la Comunicación Social (UBA). 


\section{Bibliografía}

Baladron, M. (2018). "El Plan Nacional de Telecomunicaciones Argentina Conectada (2010-2015) en el marco de las políticas públicas de universalización del acceso a internet" (tesis de posgrado). Bernal: Universidad Nacional de Quilmes (UNQ). Disponible en: <https://ridaa.unq.edu.ar/handle/ $20.500 .11807 / 788>$. Consultado el 3 de enero de 2020.

Belli, L. (ed.) (2017). Community Networks: The Internet by the People, for the People. Official Outcome of the UN IGF Dynamic Coalition on Community Connectivity. Rio de Janeiro: Escola de Direito do Rio de Janeiro da Fundação Getulio Vargas.

Deursen, A. van y Dijk, J. van (2010). "Internet Skills and the Digital Divide". New Media $y$ Society, 13(6), pp. 893-911.

Díaz, M. (2019). "Políticas habitacionales y urbanismo neoliberal: La intervención estatal en la Villa 20, Argentina (1984-2018)". Revista de Urbanismo, 40, pp. 1-19.

García-Canclini, N. (2004). Diferentes, desiguales y desconectados: Mapas de la interculturalidad. Barcelona: Gedisa.

Gendler, M. A.; Méndez, A.; Samaniego, F. y Amado, S. (2018). "Uso, apropiación, cooptación y creación: Pensando nuevas herramientas para el abordaje de la Apropiación Social de Tecnologías". En: Lago-Martínez, S.; Álvarez, A.; Gendler, M. y Méndez, A. (eds.). Acerca de la apropiación de tecnologías. Teoría, estudios y debates. Buenos Aires: Rada Tilly, Ediciones del Gato Gris, pp. 49-60.

Gil-Juárez, A.; Vitores, A.; Feliu, J. y VallLlovera, M. (2011). "Brecha digital de género: Una revisión y una propuesta". Revista Teoría de la Educación: Educación y Cultura en la Sociedad de la Información. 12 (2). Disponible en:<http:// campus.usal.es/ revistas_trabajo/index.

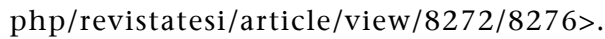
Consultado el 30 de marzo de 2020.

Gray, T. J.; Gainous, J. y Wagner, K. M. (2016). "Gender and the Digital Divide in La- tin America". Social Science Quarterly, 98(1), pp. 326-340.

Hilbert, M. (2011). "Digital Gender Divide or Technologically Empowered Women in Developing Countries? A Typical Case of Lies, Damned Lies, and Statistics". Women's Studies International Forum, 34(6), pp. 479-489.

Huerta, E. V. y Baca, C. F. (2018). "Techio comunitario: caminos hacia un programa de formación en comunicación indígena". Revista NuestrAmérica, 6 (12), pp. 22-35.

Lago-Martínez, S.; Méndez, A. y Gendler, M. (2017). "Teoría, debates y nuevas perspectivas sobre la apropiación de tecnologías digitales". En: Cabello, R. y López, A. (eds.). Contribuciones al estudio de procesos de apropiación de tecnologías. Buenos Aires: Rada Tilly, Ediciones del Gato Gris, pp. 75-86.

Monje, D.; Rivero, E.; Guzmán, F.; Martín, J.; Zanotti, J. G.; Giménez, M. E. y Villalba, V. (2017). "Otro territorio. Impacto de la convergencia periférica en el sector audiovisual de la Economía Social y Solidaria en Argentina". En: Cabral, A.; Bolaño, C.; Araujo. D.; Andacht, F. y Paulino F. (eds.). Nuevos conceptos y territorios en América Latina. Brasil: ALAIC, pp. 199-229.

Morales, S. (2017). "Imaginación y 'software': Aportes para la construcción del paradigma de la apropiación". En: Cabello, R. y López, A. (eds.). Contribuciones al estudio de procesos de apropiación de tecnologías. Buenos Aires: Rada Tilly, Ediciones del Gato Gris, pp. 39-52.

Motta, J. y Almansi, F. (2017). “Gestión y planificación pro proceso-proyecto para el mejoramiento de villas y asentamientos de gran escala. El caso de la Re-Urbanización de Villa 20 en la CABA". Medio Ambiente y Urbanización, 86(1), pp.145-168.

Pang, N. y Woo, Y. T. (2020). "What about WhatsApp? A Systematic Review of WhatsApp and Its Role in Civic and Political Engagement". First Monday, 25(12). 
Sandvig, C. (2004). "An Initial Assessment of Cooperative Action in Wi-Fi Networking". Telecommunications Policy, 28, pp. 579-602.

Trudel, D. y Tréguer, F. (2016). "Alternative Communications Networks Throughout History". NetCommons. Disponible en: $<$ https://netcommons.eu/sites/default/files/ d5.1_history_v1.1.pdf $>$. Consultado el 15 de marzo de 2018.
UIT (Unión Internacional de las Telecomunicaciones). (2019). "ICT Facts and Figures 2017". Disponible en: <https://www.itu.int/en/ ITU-D/Statistics/Documents/facts/FactsFigures 2019.pdf $>$. Consultado el 3 de enero de 2020.

Vergés-Bosch, N. (2012). "De la exclusión a la autoinclusión de las mujeres en las TIC. Motivaciones, posibilitadores y mecanismos de autoinclusión". Athenea Digital, 12(3), pp. 129-150. 